\title{
le piézocône \\ améliorations apportées à la reconnaissance des sols
}

\author{
the piezocone \\ improvement in the soil investigation
}

\author{
L. PAREZ \\ Président d'Honneur du C.F.M.S." \\ R. FAURIEL \\ Ingénieur à SOL ESSAIS ${ }^{* *}$
}

Rev. Franç. Géotech. nं 44, p.p. $13 \cdot 27$ (juillet 1988)

\section{Résumé}

Lorsqu'un capteur de pression est incorporé dans un pénétromètre statique électrique, cet ensemble est appelé piézocône.

Les auteurs donnent l'état des connaissances de cet essai relativement récent. Ils décrivent les précautions à prendre pour faire des mesures fiables de la pression interstitielle générée par la pénétration du cône. Ils montrent l'intérêt du piézocône pour obtenir des profils stratigraphiques précis, pour connaître la résistance au cisaillement des argiles, pour apprécier le potentiel de liquéfaction au séisme de couches sableuses, et pour faire des prédictions de temps de consolidation de couches compressibles chargées in situ (courbe $K_{h}$ en fonction de $t_{50}$ ).

\section{Abstract}

When a pressure transducer is incorporated into standard electric friction cone, this device is called a piezocone.

The authors give the state of the art of that relatively recent test. They describe the care to obtain trusted values of the pore pressure generated by the penetration of the cone.

They show the ability to have accurate soil classification from piezocone data, to obtain undrained shear strength in clayed layers, to estimate liquefaction potential of sandy layers and to forecast the consolidation time after an in situ loading (curve $K_{\mathrm{h}}$ versus $t_{50}$ ).

4, place de Mexico, 75116 Paris.

"* 35, rue de la Molle, 13100 Aix-en-Provence. 


\section{GÉNÉRALITÉS}

L'installation d'un capteur de pression dans une pointe de pénétromètre statique a été un grand progrès dans les essais in situ: elle a permis de mesurer, à tout moment de la pénétration, la valeur de la pression interstitielle générée par l'enfoncement du pénétromètre.

Les premières mesures de ce genre ont été publiées par JANBU et SENNESET en 1974 à Stockholm au cours du Symposium Européen ESOPT, Des recherches étaient en cours dans le même temps à SOL ESSAIS. La figure 1 montre un diagramme d'essai réalisé à Bayonne en début décembre 1974. Ces premiers résultats ont été publiés par L. PAREZ, M. BACHELIER et B. SECHET au Congrès Européen de Vienne en 1976. Depuis cette date, et en particulier à partir de 1981, de nombreuses publications sont apparues en provenance des pays suivants: Norvège, Suède, Hollande, Italie, Canada, Etats-Unis, Afrique du Sud, Japon. Rapidement, un accord est intervenu entre anglophones et francophones pour baptiser l'appareil : " piézocône».

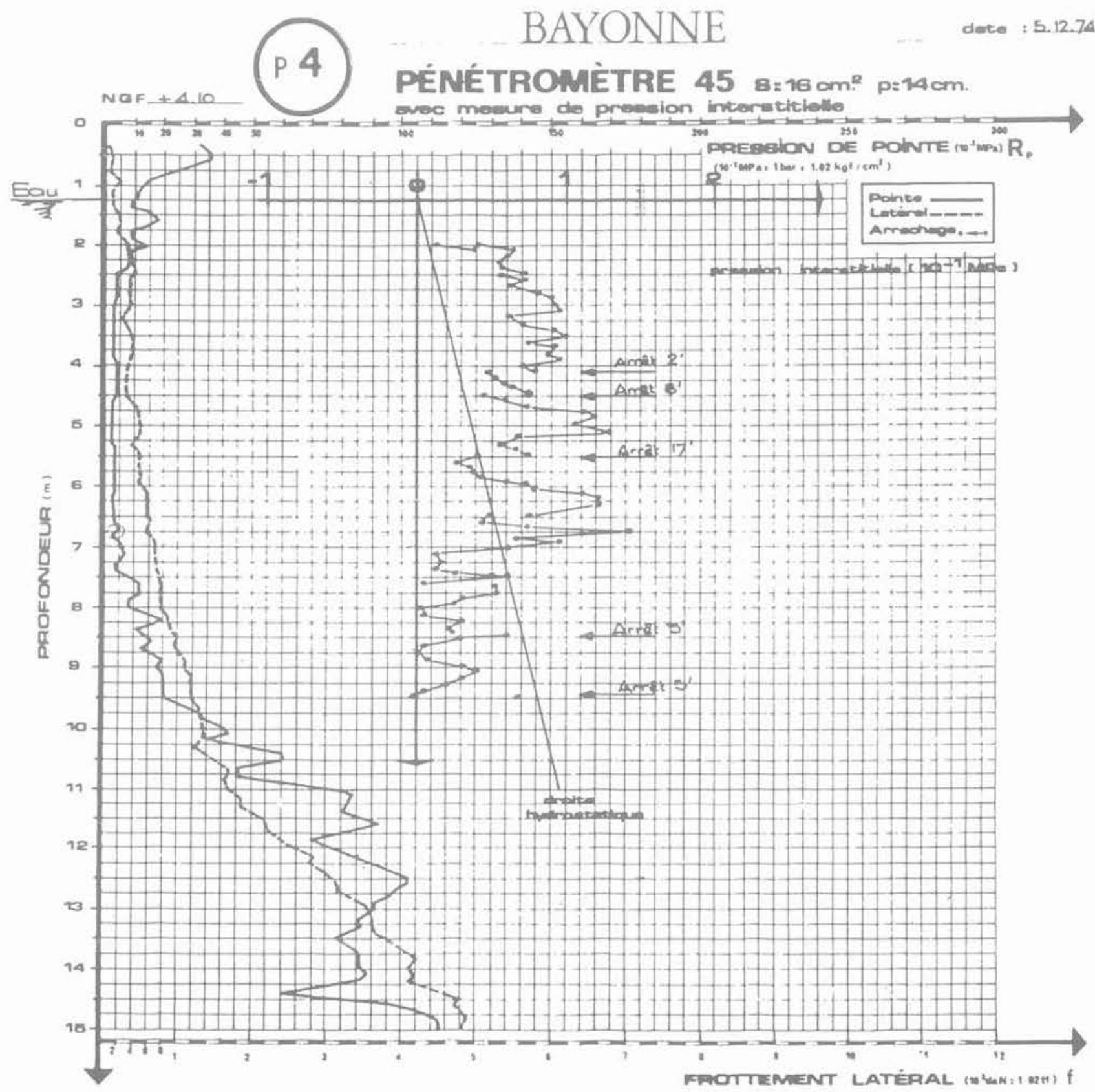

Fig. 1. - Graphique de piézocône de 1974

Fig. 1. - Piezocone graph (1974) 
La figure 2 montre la coupe du piézocône SOL ES. SAIS. (diamètre $36 \mathrm{~mm}$ ou $45 \mathrm{~mm}$ ).

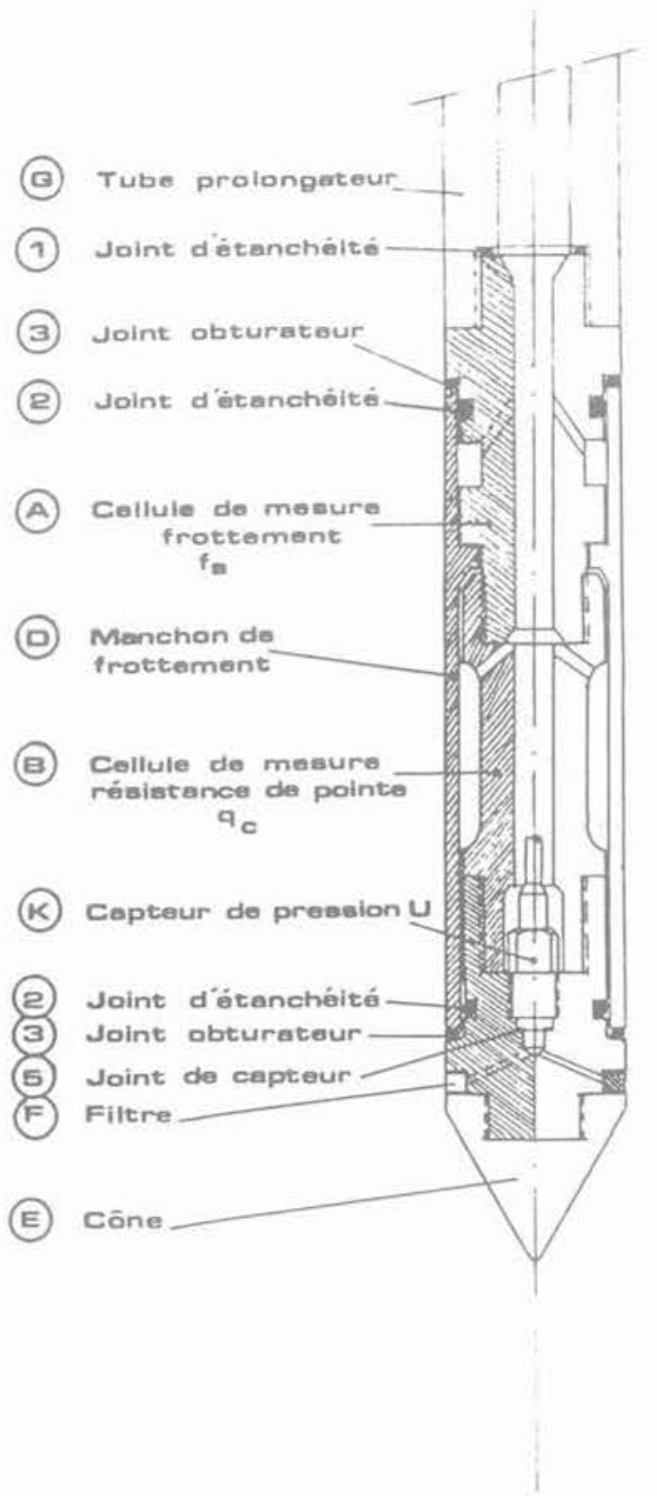

Fig. 2. - Piézocône PAREZ

Fig. 2. - PAREZ Piezocone

La figure 3 montre les courbes obtenues avec un piézocône comportant un manchon de frottement: $q_{e}$; $\mathrm{u}, \mathrm{f}_{\mathrm{s}}, \mathrm{F}_{\mathrm{R}}, \Delta \mathrm{u} / \mathrm{q}_{\mathrm{c}}$

\section{MESURE DE U}

Dans les sols saturés, l'introduction de la pointe d'un pénétromètre statique provoque un cisaillement qui s'accompagne d'une variation $\Delta \mathrm{u}$ de la pression inters. titielle: $\Delta u>0$ dans les sols contractants et $\Delta u<0$ dans les sols dilatants.
Les valeurs obtenues pour $\Delta \mathrm{u}$ sont influencées par un certain nombre de facteurs dont les uns sont liés au sol (compacité - teneur en fines - perméabilité rapport de surconsolidation, etc.) et les autres dépendent de l'appareil ; ce sont, principalement:

- la position du filtre de prise de pression,

- la vitesse de pénétration,

- la vitesse de réponse du capteur et de son système de mesure,

- la qualité de la saturation de la chambre de mesure (y compris le filtre).

\subsection{Position du filtre de prise de pression}

La figure 4, due à ROBERTSON, montre les différentes positions des bagues-filtres servant à la prise de pression. Les croquis $n^{c} 1$ et 2 ne correspondent pas à la recommandation européenne car l'angle du cône n'est pas de $60^{\circ}$.

Des mesures comparatives de $\Delta u$ ont été faites dans des sites argileux à peu près homogènes aux USA et au Canada (fig. 5).

NORMALIZED EXCESS PORE PRESSURE, $\triangle U /(\Delta U)_{S H}$

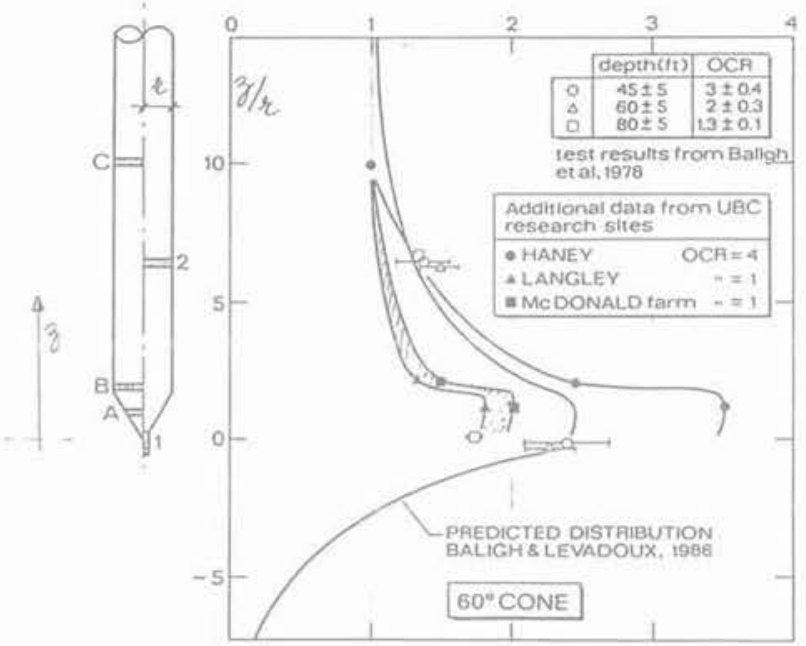

Fig. 5. - Comparaison entre pressions interstitielles mesurées et calculées.

Fig. 5. - Comparison between measured and predicted pore pressures

Pour les argiles normalement consolidées les valeurs de $\Delta u$ mesurées en $A$ sont environ $35 \%$ plus fortes que celles mesurées en B (faisceau de gauche), ce rapport passe à $44 \%$ pour une argile assez surconsolidée $(\mathrm{OCR}=4-$ courbe de droite)

De même, au Japon, SUGAWARA et CHIKARAISHI (fig. 6) ont trouvé une augmentation de $40 \%$ entre les valeurs mesurées aux mêmes points (qu'ils appellent B et $\mathrm{C}$ ) sans indiquer de rapport de surconsolidation pour leur site argileux. 
P2-
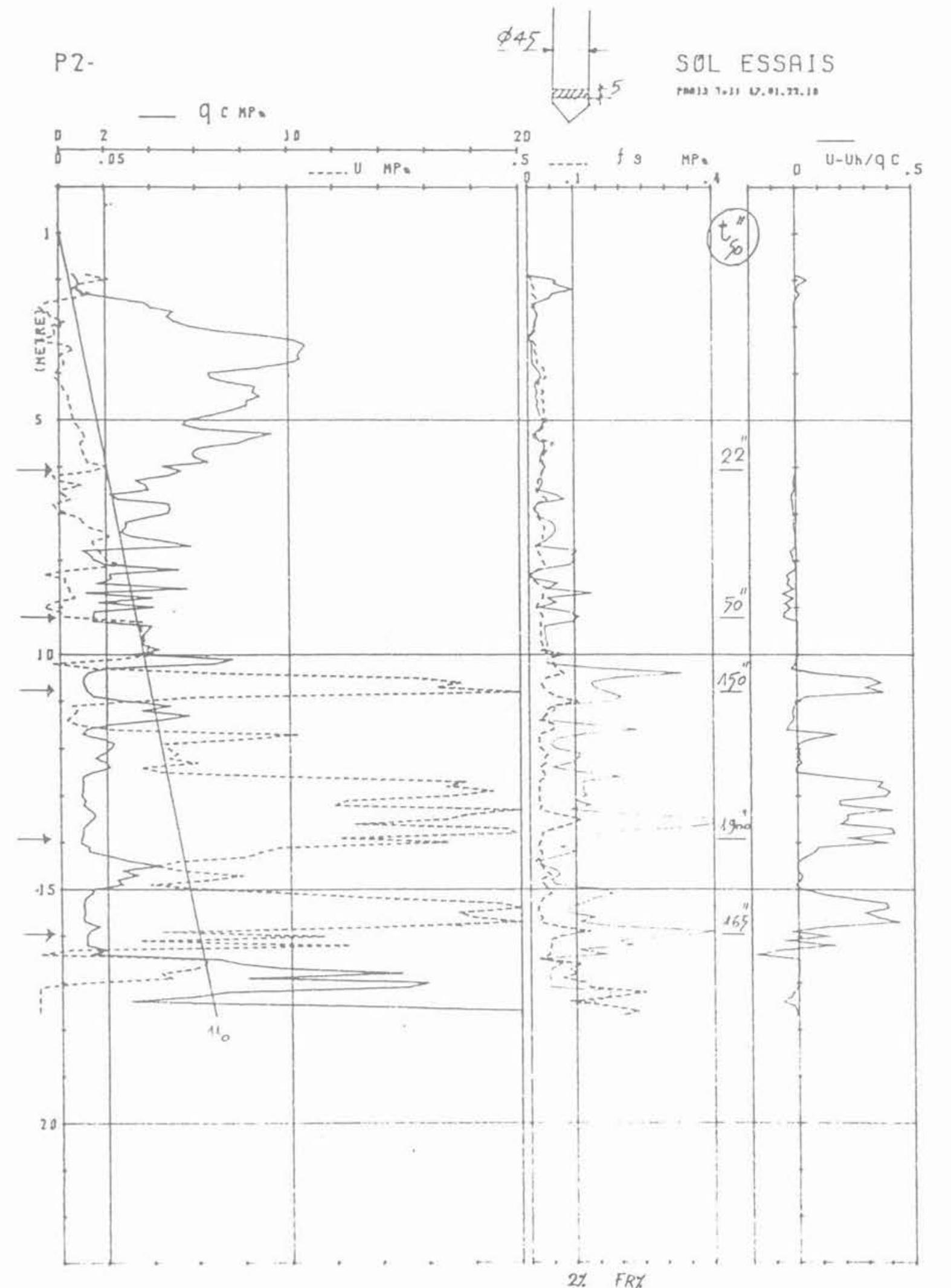

Fig. 3. - Exemple d'un graphique actuel.

Fig. 3. - Example of a current graphic. 

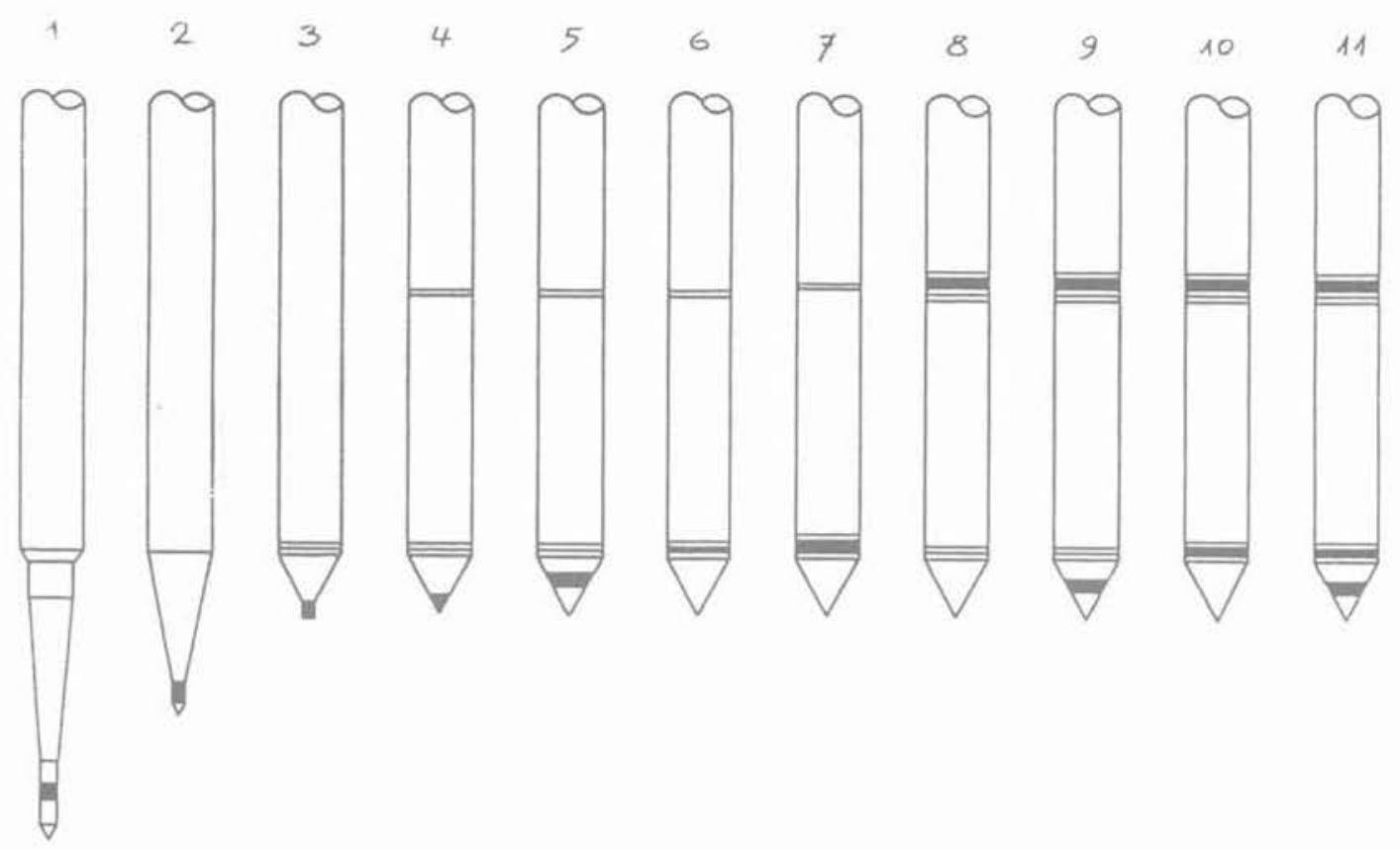

Fig. 4. - Différents piézocönes montrant différentes positions de prise de pression (selon Robertson).

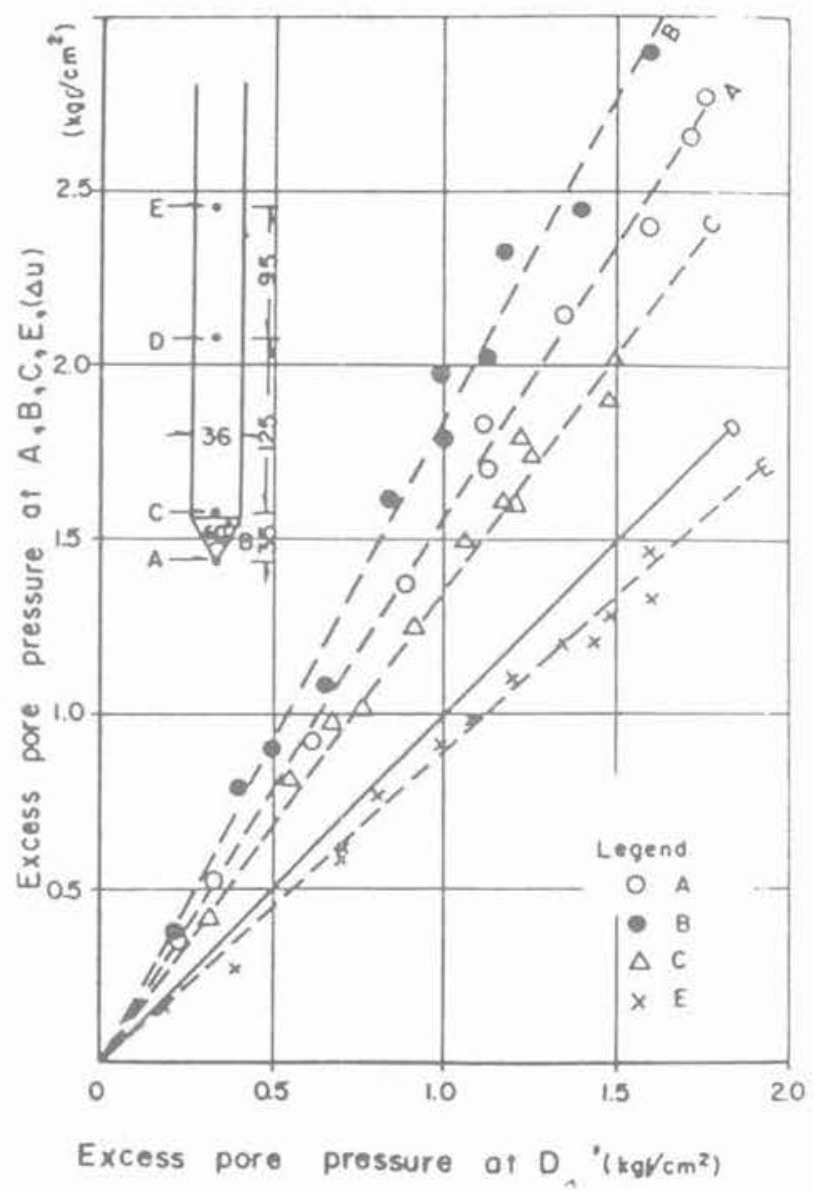

Fig. 6, - Pression interstitielle en différents points rapportée à celle au point $D$.

Fig. 6. - Pore pressure at different locations and at D
Nous avons fait une comparaison récente dans un site d'alluvions modernes limono-argileuses et nous n'avons pas trouvé de différence nettement sensible entre les mesures avec prise de pression sur le cône ou prise de pression juste au-dessus du cône. Seuls les $t_{50}$ varient d'une façon importante.

Dans les couches sableuses compactes, un filtre situé au-dessus du cône permet de mesurer des $\Delta u$ négatifs : si le filtre se trouve sur le cône, dans cette zone où les contraintes normales sont très fortes souvent la dila. tance ne peut se produire, et alors on n'enregistre pas de $\Delta u$ négatif.

La tendance actuelle est de positionner la bague-filtre de prise de pression juste au-dessus du cône, elle est ainsi moins sujette aux destructions, elle permet de mesurer une large gamme de surpressions interstitielles (depuis les négatives jusqu'aux fortement positives), elle est au meilleur endroit pour appliquer la correction de $\mathrm{q}$ dont nous parlerons plus loin. Evidemment, cette position ne permet pas d'obtenir les valeurs maximales de $\mathrm{U}$ mais elle permet la mesure des $\Delta \mathrm{u}$ faibles ou négatives dans les limons et sables fins et des fortes valeurs positives dans les argiles.

\subsection{Vitesse de pénétration}

Le standard européen pour le pénétromètre statique est de $2 \mathrm{~cm} / \mathrm{seconde}$ pour la vitesse de fonçage, avec une tolérance de $\pm 0,5 \mathrm{~cm} / \mathrm{s}$. On admet généralement qu'à cette vitesse on est en condition drainée dans le sable (pas totalement, nous le verrons plus loin) et en condition non drainée dans l'argile.

CANOU, avec le minipiézocône du Sermès, a montré, dans un sable assez perméable, l'influence de la vitesse 
de fonçage sur la pression interstitielle générée au fonçage, (fig. 7) aussi nous avons demandé à la Commission de Standardisation internationale de réduire la fourchette de tolérance de vitesse, pour le piézocône, à $\pm 0,2 \mathrm{~cm} / \mathrm{s}$.

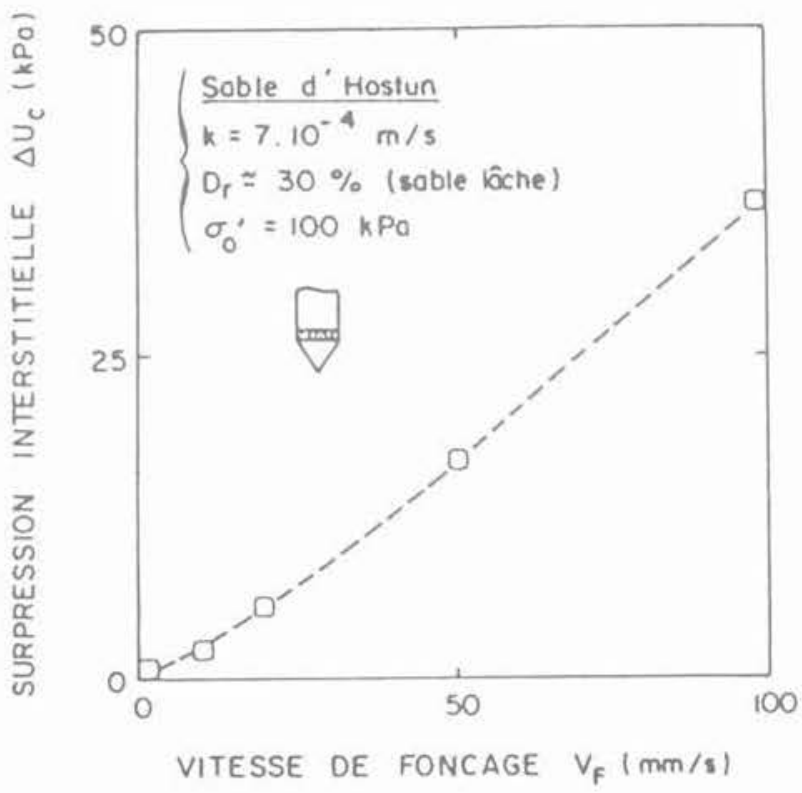

Fig. 7. - Influence de la vitesse de fonçage sur la surpression interstitielle.

Fig. 7. - Rate of pushing and excess of pore pressure.

\subsection{Vitesse de réponse du capteur}

L'ensemble du système de mesure de la pression interstitielle comprend:

- la bague-filtre,

- les canaux et la chambre de mesure,

- le capteur de pression,

- la chaîne de lecture et d'enregistrement.

L'utilisation des micro-ordinateurs avec carte de saisie ultra-rapide a permis une précision bien plus grande que l'emploi des tables traçantes dont l'inertie peut camoufler les pointes des valeurs de $\mathrm{u}$ et $\mathrm{q}_{\mathrm{r}}$. De plus on obtient automatiquement le calcul de $\Delta \mathrm{u} / \mathrm{q}_{\mathrm{c}}$ ou mieux de $\Delta \mathrm{u} /\left(\mathrm{q}_{\mathrm{T}}-\sigma_{\mathrm{vo}}\right)$.

Les premiers capteurs de pression utilisés étaient des capteurs à corde vibrante dont la membrane était assez déformable ce qui offrait l'inconvénient de donner un retard à la réponse et d'écrêter les valeurs maximales dans les sols peu perméables.

Les capteurs utilisés maintenant sont basés sur l'utilisation de membranes piézorésistives très peu déformables et pourtant très précises. Ils sont compensés pour les variations de température.

Reste le problème de la déformabilité du volume du liquide qui transmet la pression de l'extérieur du piézocône à la membrane. Supposons que ce liquide soit de l'eau (parfaitement désaérée, bien entendu) sa compressibilité sous un accroissement de pression $\Delta p$ (en $\mathrm{kPa}$, entre 0 et $1.000 \mathrm{kPa}$ ) est de lordre de $\Delta \mathrm{v} / \mathrm{v}$ $=5.10^{-7} \Delta \mathrm{p}$. Si le volume de liquide est de $300 \mathrm{~mm} 3$ sa variation sous un accroissement de pression de $100 \mathrm{kPa}$ sera $\Delta v=15 \cdot 10^{-3} \mathrm{~mm} 3$. La surface latérale extérieure du filtre étant d'environ $500 \mathrm{~mm} 2$, P'épaisseur de la lame d'eau qui doit y pénétrer est de l'ordre de $3.10^{-5} \mathrm{~mm}=3.10^{-8} \mathrm{~m}$.

Si le sol au contact de la bague est de largile avec une perméabilité de l'ordre de $3 \cdot 10^{-10} \mathrm{~m} / \mathrm{s}$, le gradient étant très fort, il faudra entre 0,3 et 1 seconde pour que le capteur traduise en totalité cette variation $\Delta \mathrm{u}=$ $100 \mathrm{kPa}$.

Si le volume de la chambre de mesure est le triple, comme sur certains appareils, le temps sera de 3 secondes et l'appareil aura pénétré de 6 centimètres, dans une couche peut-être différente...

On voit donc tout l'intérêt qu'il y a à miniaturiser au maximum la chambre de mesure, y compris la bague poreuse dont le diamètre extérieur doit rester néanmoins égal au diamètre de base du cône.

\subsection{Qualité de la saturation de la chambre de mesure}

La saturation du piézocône consiste à éliminer toutes les bulles d'air présentes dans les éléments filtrants (pierres poreuses) et la chambre de mesure où aboutit le capteur de pression interstitielle.

On conçoit aisément l'importance de cette opération puisque la présence d'air, fortement compressible, nuirait grandement à la qualité des mesures, anéantissant du même coup les efforts consentis sur le plan de la miniaturisation de la chambre de mesure ainsi que sur celui de la valeur du capteur proprement dit.

Afin de saturer convenablement le piézocône SOL ESSAIS, nous avions dans un premier temps suivi la méthode préconisée par la Société FUGRO.

Cette technique impose de placer la pointe du piézocône dans une petite cellule en plexiglas à l'intérieur de laquelle on fait le vide durant une quarantaine de minutes puis que l'on remplit progressivement d'eau désaérée (figure 8).

Lorsque cette opération était terminée, au bout d'une heure trente environ, nous pensions que la saturation du piézocône était correcte.

Malheureusement, lors des premiers essais * in situ * réalisés, nous avons observé un phénomène caractérisant nettement un défaut de saturation.

En effet, lors des arrêts de pénétration destinés à enregistrer la dissipation de surpression interstitielle, le maximum de celle-ci était obtenu seulement quelques minutes après l'arrêt dans les sols argileux.

Ce retard, pour lequel le capteur ne pouvait être mis en cause, nous a conduit à mettre en place une série d'expérimentations destinées à déterminer un mode de saturation plus efficace. 
SATURATIOA ELEMENTS FILTRANTS EI CHAMBRE

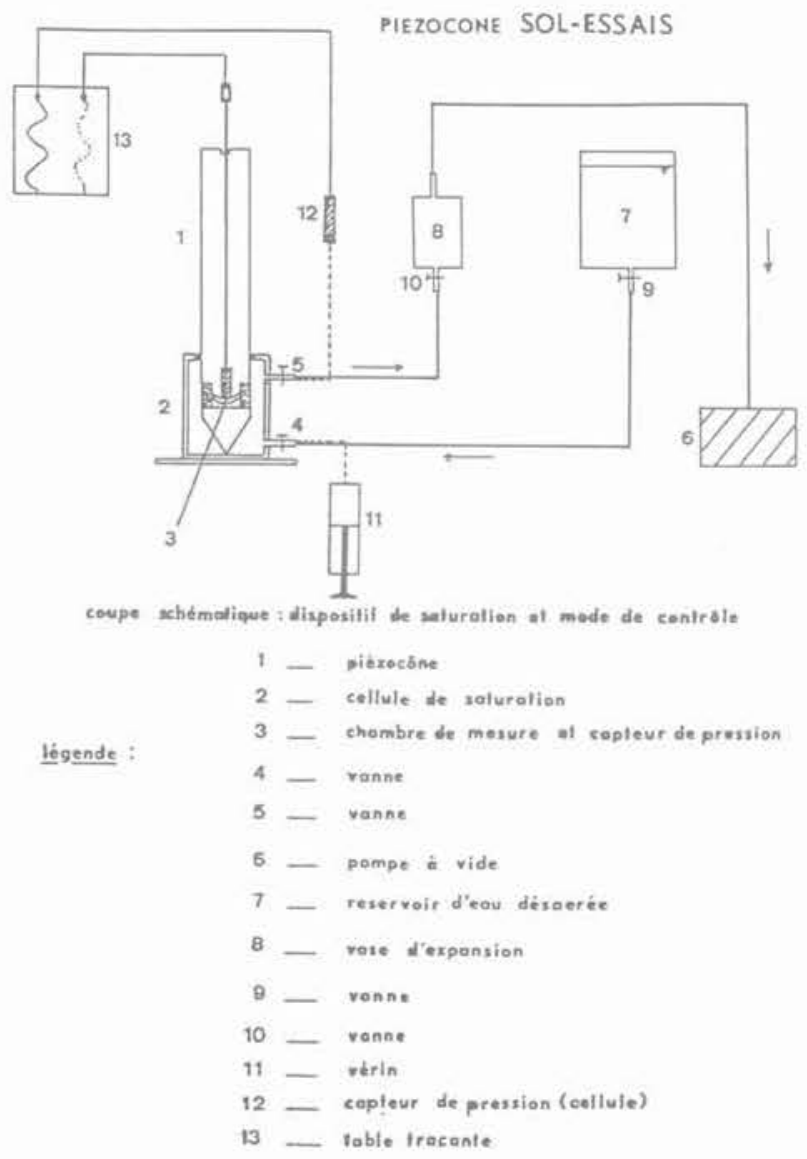

Fig. 8. - Dispositif de saturation et mode de contrôle. Fig 8. - Saturation device and testing method.

Pour cela, nous avons équipé la cellule en plexiglas avec un système permettant d'appliquer des variations de pression à lintérieur de celle-ci (remplie d'eau bien évidemment).

Nous avons également installé un capteur de pression interstitielle sur cette cellule afin de mesurer les variations de pression appliquées.

Enfin, nous avons enregistré, en parallèle, sur une table traçante les courbes représentant ces sollicitations et les réponses du piézocône, placé dans la cellule (figure 8 . en pointillés).

La figure 9-A illustre le type de graphiques obtenus dans le cas où le piézocône est saturé avec la méthode décrite auparavant.

On remarque une assez bonne concordance entre les sollicitations (capteur cellule) et les réponses du piézocône, autant en ce qui concerne les retards de réponse que les amplitudes de pression: cela tendrait donc à démontrer que ce mode de saturation est efficace.

Cependant, lorsque nous avons testé le piézocône sans l'avoir saturé au préalable nous avons également noté une parfaite similitude des deux courbes.
Il fallait dont tenter de limiter les échanges d'eau entre la chambre de mesure et le milieu environnant qui atténuent les effets néfastes d'une mauvaise saturation lorsqu'ils sont très importants (ce qui était bien le cas lors de nos expérimentations) mais qui sont très réduits dans les sols.

Afin de simuler au mieux ce comportement $\alpha$ in situ $~$. nous avons disposé une membrane en caoutchouc sur la pierre poreuse du piézocône, empêchant ainsi tout transfert d'eau entre l'intérieur et l'extérieur de la chambre de mesure (cas extrême).

Dans ce cas, labsence de saturation apparait nettement (figure 9-B). Le déphasage entre les sollicitations appliquées et les réponses du piézocône est très important et illustre bien le danger d'une mauvaise saturation dans le cadre des mesures de pression interstitielle.

Après nous être assurés que la méthode de saturation initialement adoptée ne convenait pas (figure 9-C), bien que meilleure qu'une absence totale de saturation, nous avons développé une série d'expérimentations nous permettant de définir un mode de saturation « optimal », associant une excellente qualité de mesures à des contraintes économiques réduites (matériels et temps de saturation peu importants).

$\mathrm{Ce}$ mode opératoire consiste à saturer séparément l'élément filtrant (en faisant bouillir les pierres poreuses, par exemple), et la chambre de mesure du piézocône au moyen de la cellule en plexiglas. La durée totale de ces opérations, simultanées, est de l'ordre de 2 heures.

On assemble ensuite les deux parties dans un bac d'eau désaérée avant de réaliser les essais * in situ ».

Lors du test de cette technique de saturation au moyen de l'expérimentation précédemment décrite, nous avons obtenu d'excellentes concordances entre les sollicitations appliquées et les réponses du piézocône (figure 9-D).

On remarque notamment que le délai de réponse ne dépasse pas 5 centièmes de seconde et que les amplitudes de pression ne different que de $2,5 \mathrm{kPa}$, ce qui compte tenu des imprécisions liées au type d'enregistrement (analogique et graphique) est parfaitement convenable.

Nous terminerons en rappelant que, lors de cette expérimentation, notre but n'était pas de définir quantitativement et de façon très précise la qualité de la saturation selon le mode opératoire utilisé, mais de définir celui-ci dans le respect de critères économiques et techniques simples.

Dans cette optique, nous considérons avoir atteint l'objectif fixé, ainsi que l'ont d'ailleurs confirmé les essais réalisés * in situ * par la suite.

Au problème de la parfaite saturation préalable s'ajoute celui du risque de désaturation pendant la mise en place de l'appareil jusque sous le niveau de la nappe.

On peut lire sur le texte de FRANKLIN et COOPER publié à Stockholm en 1981 que l'élément poreux qu'ils utilisent est très fin et «suffisamment perméable pour donner une réponse rapide de la cellule de 


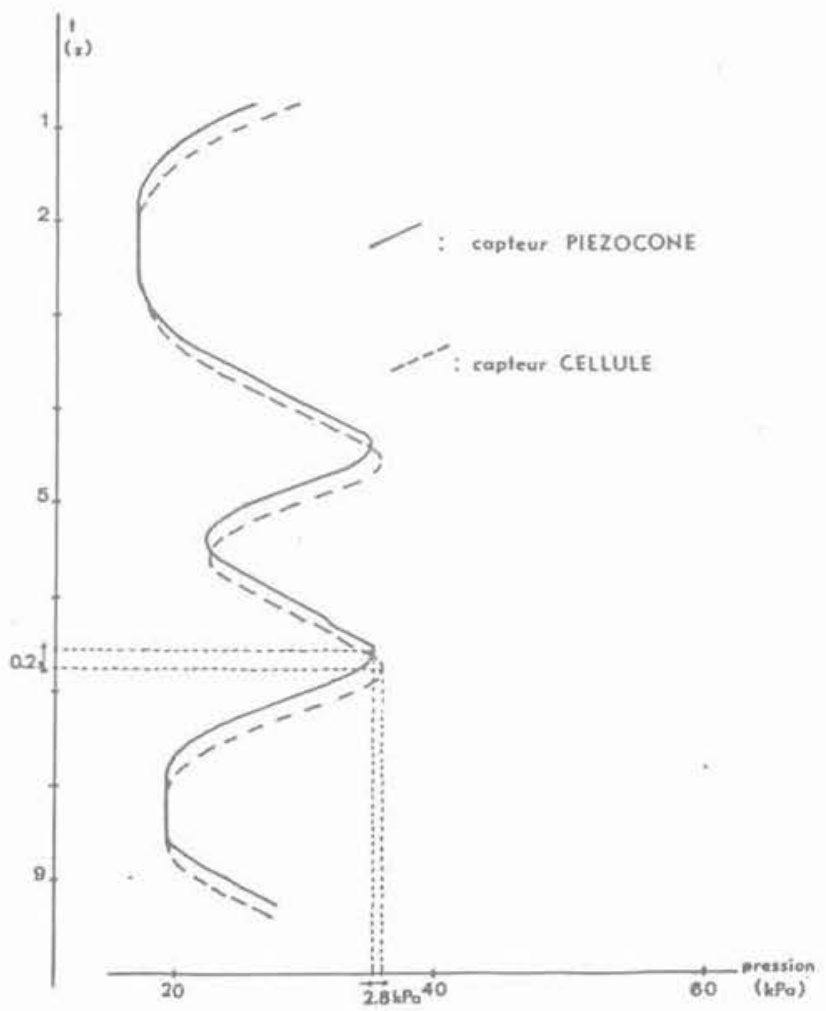

a)

SATURATION FLEMENTS FILTRANTS IT CHAMBRE

MEZOCONE SOL-ESSAIS

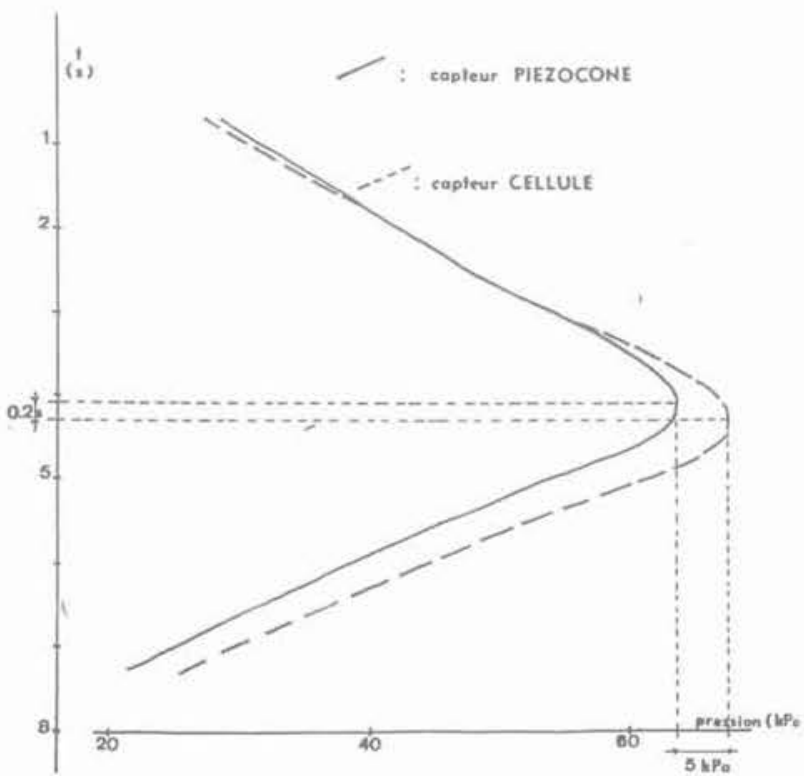

c)

Fig. 9. - A à D Mise en charge de la cellule et réponse du piézocône

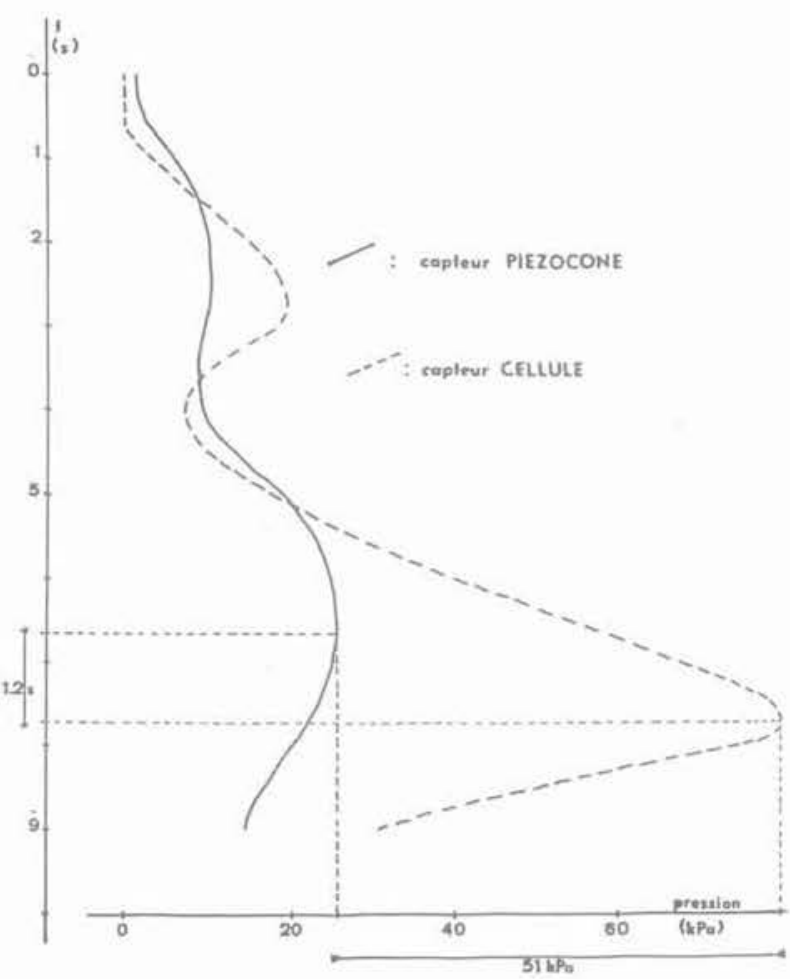

b)

SATURATION FLEMENTS HLTRANTS ET CHAMBRE

PEZOCONE SOL-ESSAIS

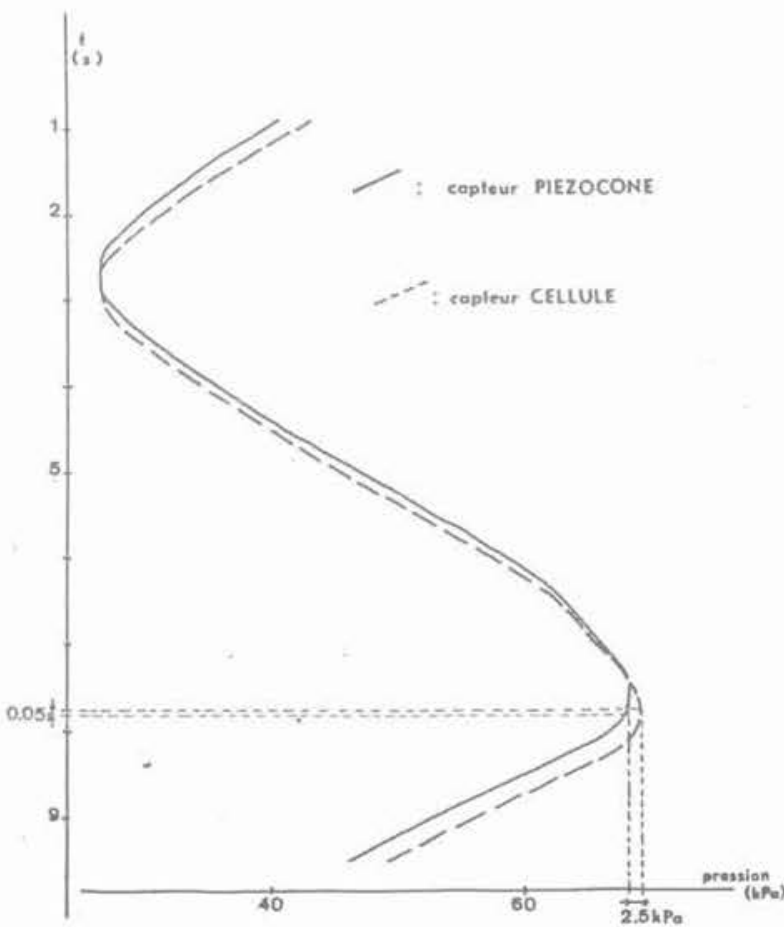

d)

Fig. 9. - A at D Loading of the cell and piezocone answer 
pression et pour offrir une résistance suffisante à l'entrée de l'air durant les courtes périodes où le cône est exposé à l'air ». Ainsi, de trop nombreux expérimentateurs prennent le risque de désaturation partielle de l'élément poreux en commençant la pénétration, sans précaution particulière, depuis la surface du sol alors que la nappe se trouve à plusieurs mètres de profondeur. La hauteur d'ascension capillaire n'est pas toujours suffisante, et, comme l'indique ROBERTSON, des argiles raides surconsolidées peuvent donner de la suc. cion. Il en est de même pour les sables au-dessus de la densité critique. La seule méthode satisfaisante consiste à faire un avant-trou jusqu'à quelques décimètres sous la nappe et à y descendre le piézocône protégé par une capote remplie d'eau désaérée, capote qui se rompt dès le début de la pénétration.

\section{CORRECTION DE $\mathrm{q}_{\mathrm{c}}$ : (fig. 10)}

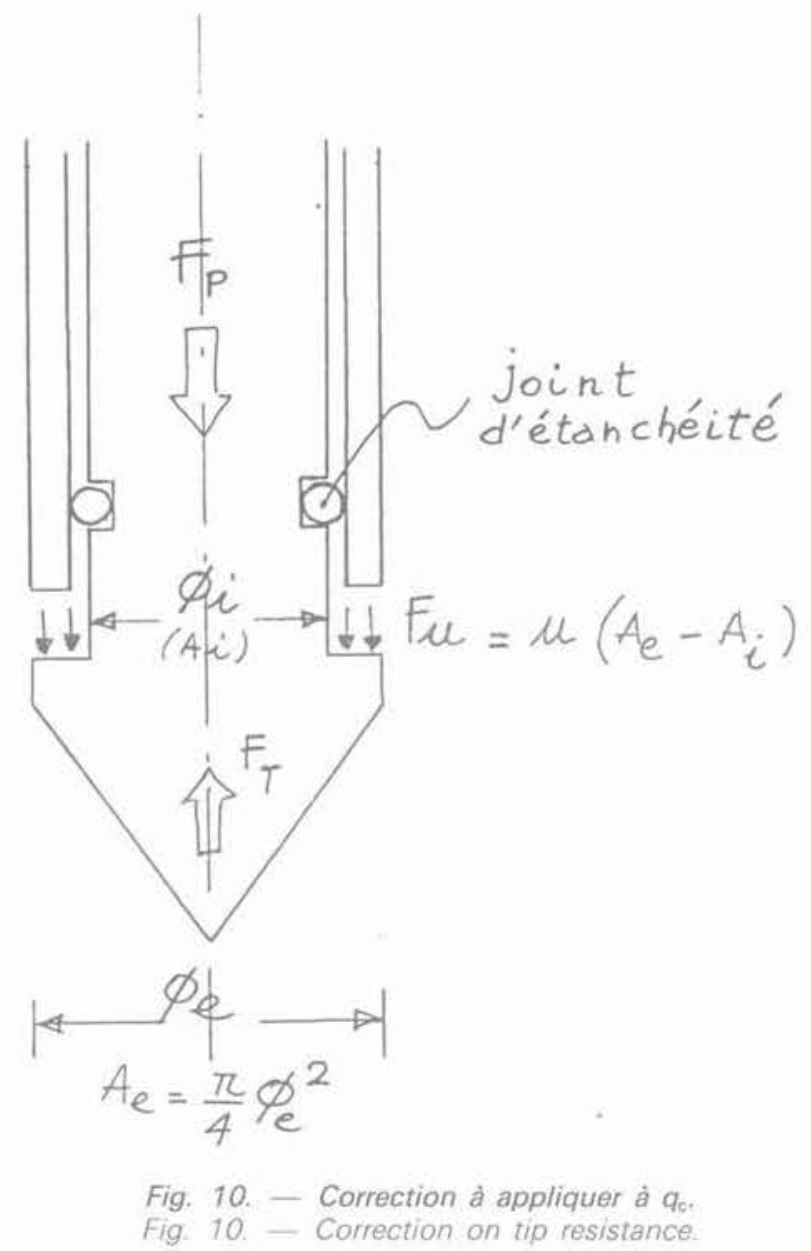

Sous la nappe, l'effort vrai ou total sous le cône $F_{T}$ est égal à l'effort de pointe mesuré $F_{p}$ augmenté de l'effort $F_{u}$ dû à l'action de la pression interstituelle $u$ sur la section annulaire de l'appareil:

$$
F_{T}=F_{P}+F_{u} \text { avec } F_{u}=u\left(A_{e}-A_{i}\right)
$$

en divisant par $\mathrm{A}_{\mathrm{e}}$ (surface de base du cône)

$$
\mathrm{q}_{\mathrm{T}}=\mathrm{q}_{\mathrm{c}}+\mathrm{u}\left(1-\frac{\mathrm{A}}{\mathrm{A}_{\mathrm{e}}}\right)=\mathrm{q}_{\mathrm{c}}+\mathrm{u}(1-\mathrm{a})
$$

Cette correction proposée par BALIGH en 1981 et reprise par CAMPANELLA, peut être importante dans les argiles molles où $q_{c}$ est faible et $U$ relativement fort.

Le coefficient 1 - a vaut 0,23 pour le piézocône SOL ESSAIS de $45 \mathrm{~mm}$ de diamètre et 0,31 pour celui de $36 \mathrm{~mm}$. On voit ici lintérêt de la mesure de $U$ avec une bague située au-dessus du cône, c'est-à-dire dans la zone où il faut faire la correction.

Lorsque les deux extrémités du manchon de frottement sont identiques, la correction à faire ne devrait porter que sur la différence des pressions interstitielles régnant aux extrémités du manchon, mais cette correction n'est jamais faite.

\section{CLASSIFICATION DES SOLS PROFIL STRATIGRAPHIQUE}

Le piézocône donne, en fonction de la profondeur atteinte par la pointe, deux courbes: $q_{c}$ (corrigé en $q_{r}$ ) et $\mathrm{u}$. On obtient une troisième courbe, en divisant $\Delta \mathrm{u}$ (variation de $\mathrm{u}$ par rapport à la pression hydrostatique vierge) par $q_{\text {, ou }} q_{1}$ ou, mieux encore, par:

$$
q_{T}-\sigma_{v o} \text { soit } B_{q}=\frac{\Delta u}{q_{T}-\sigma_{v o}}
$$

( $\sigma_{u \text { e }}$ étant la contrainte verticale totale due au poids des terres situées au-dessus de la profondeur de mesure).

La comparaison de ces courbes et, en particulier, l'examen de la troisième indique la nature des couches successives d'une façon encore plus nette que ne le faisait le Friction Ratio (voir la figure 3).

Les sables compacts ont un $\mathrm{q}$ important et un $\Delta \mathrm{u}$ faible ou négatif donc $\Delta \mathrm{u} / \mathrm{q}_{\mathrm{c}}$ est pratiquement nul.

A l'inverse, les argiles ont un $q$ faible et un $\Delta u$ fort d'où un $\Delta \mathrm{u} / \mathrm{q}_{\mathrm{c}}$ qui va de 0,4 à 0,8 ou plus si l'argile est molle.

SENNESET et JANBU ont donné, en 1984, un diagramme que nous avons complété et modifié pour tenir compte des valeurs négatives de $\Delta u$ (fig. 11); il permet d'avoir rapidement une bonne idée de la nature des couches rencontrées en tenant compte aussi des temps de dissipation de $\Delta u$ que nous étudierons plus loin. Mais c'est seulement un guide qui ne prend pas en compte les sols surconsolidés ou très sensibles ou cimentés, et qui ne dispense pas de réaliser, sur chaque chantier, un forage carotté. 


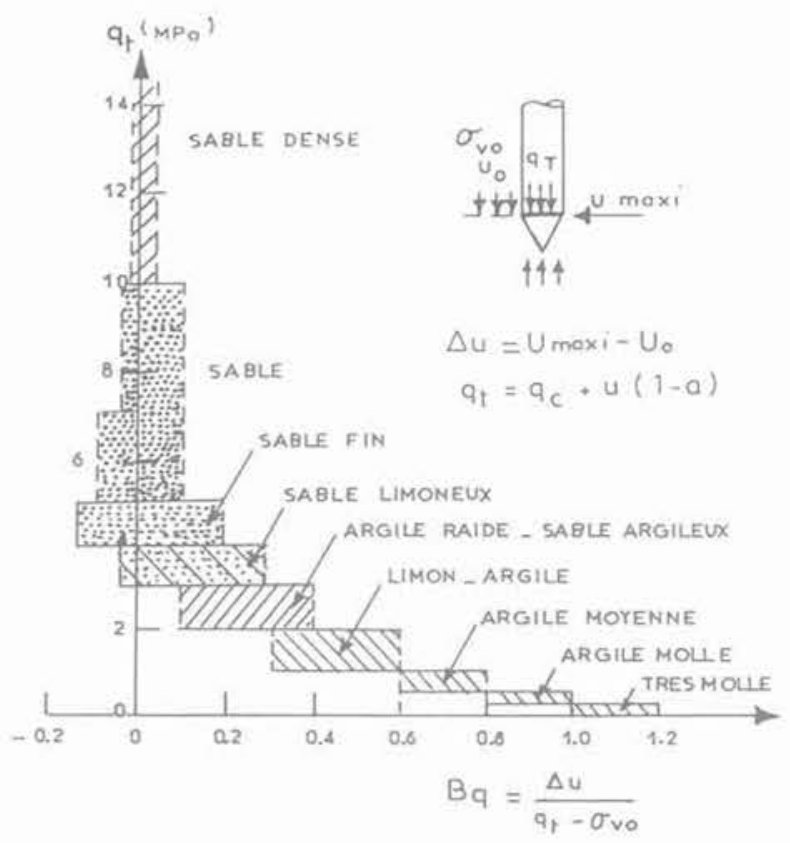

Fig. 11. - Classification des sols à partir du piézocône. Fig. 11. - Soil classification chart from piezocone data.

\section{RÉSISTANCE AU CISAILLEMENT}

\subsection{Non drainée}

Dans les sols argileux, la difficulté provient du fait que $C$ dépend du type d'essai et de la vitesse de déformation. On a ainsi bien du mal à rapprocher les résultats d'expérimentateurs différents. Pour les Nordiques, les Canadiens et les chercheurs des Etats-Unis $\mathrm{C}_{\mathrm{u}}$ est obtenu au scissomètre.

Avec le piézocône on peut parler de résistance de pointe effective $q_{T F}=q_{T}-u$ et on peut donc écrire :

$$
C_{u}=\frac{q_{T}-u}{N_{K E}}
$$

avec $\mathrm{N}_{\mathrm{KE}}$ = facteur semi-empirique que SENNESET a donné en 1982 pour $\mathrm{N}_{\mathrm{KE}}=9 \pm 3$

D'autre part, dans une couche d'argile homogène, la relation empirique $\Delta \mathrm{u}=\mathrm{N}_{\mathrm{su}} \times \mathrm{C}_{\mathrm{u}}$ est assez bien vérifiée et $\mathrm{N}_{\text {u }}$ est à peu près constant si les indices de plasticité et de liquidité restent constants.

L'emploi d'un sondage au scissomètre combiné avec des essais au piézocône semble le moyen idéal pour obtenir les variations naturelles d'une couche d'argile.

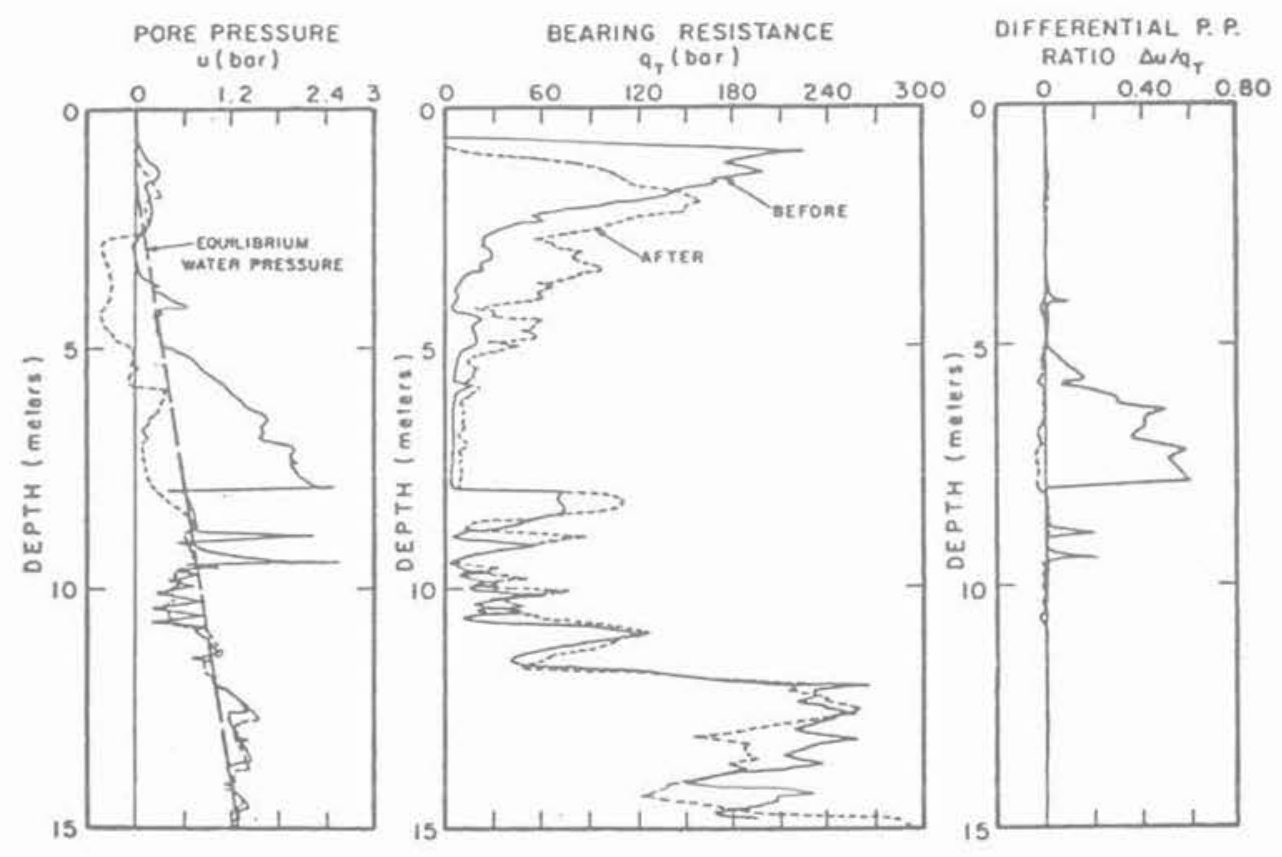

Fig. 12. - Courbes de piézocône avant et après compactage dynamique.

Fig. 12. - Piezocone logging before and after dynamic compaction. 


\subsection{Drainée}

Il n'est pas possible à l'heure actuelle de déterminer les paramètres de cisaillement en contraintes effectives $\left(C^{\prime}\right.$ et $\varphi^{\prime}$ ) à partir des résultats de la pénétration d'un piézocône. Les quelques tentatives n'ont pas abouti jusqu'ici.

\section{RÉSISTANCE A LA LIQUÉFACTION}

La figure 12 due à CAMPANELLA et al. montre les trois courbes d'essais au piézocône ( $u, q_{T}$ et $\Delta u / q_{\tau}$ ) exécutés avant et après la réalisation d'un compactage dynamique sur un site qui comportait $5 \mathrm{~m}$ de remblai hydraulique sableux sur $3 \mathrm{~m}$ de remblai hydraulique limoneux.

Avant traitement, la variation de pression interstitielle était largement positive dans la couche limoneuse alors que, après compactage dynamique, la pression interstitielle induite par la pénétration du piézocône était nettement inférieure à la pression hydrostatique. Dans la partie sableuse, elle est même devenue inférieure à la pression atmosphérique.

Du point de vue de la résistance à la liquéfaction on voit un important changement de caractéristique: le limon qui était contractant devient dilatant bien que sa résistance sous la pointe du pénétromètre $\left(q_{c}\right)$ n'ait pas augmenté beaucoup.

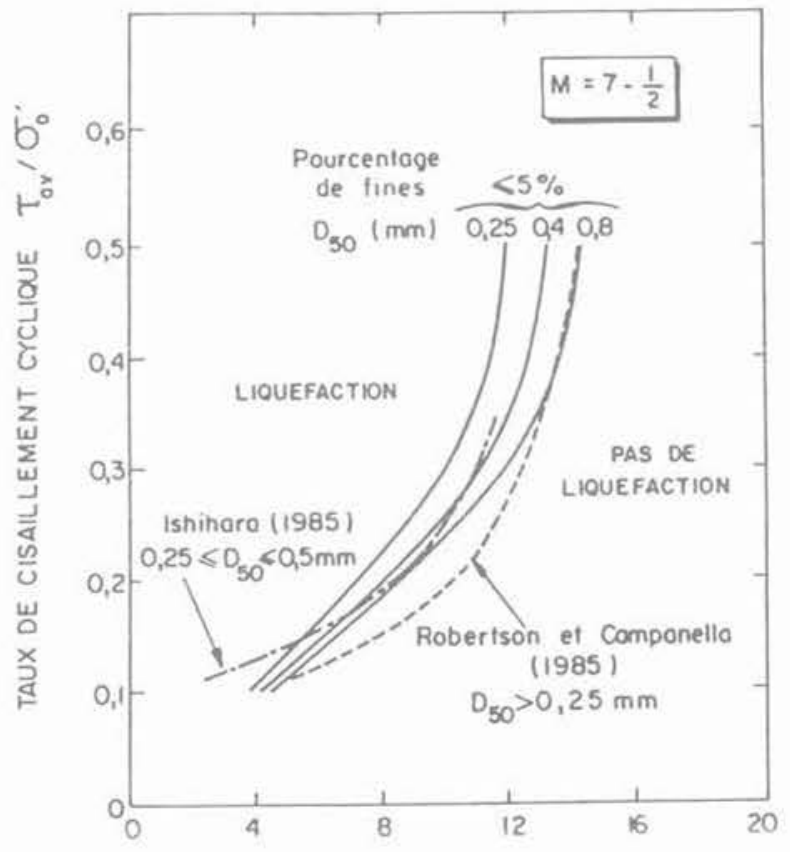

RESISTANCE DE POINTE AU PENETROMETRE $q_{c_{1}}$ (MP)

Fig. 13. - Evaluation des courbes frontières de la liquéfaction à partir du pénétromètre.

Fig. 13. - Correlation of liquefaction characteristics with CPT data.
Le Professeur SEED, en 1983 au Symposium de Paris, a montré l'intérêt du pénétromètre statique pour l'évaluation du potentiel de liquéfaction au séisme d'un site (figure 13). Il est certain que le piézocône apporte encore un plus dans ce domaine et un groupe de recherche travaille actuellement sur ce problème.

\section{CONSOLIDATION ET PERMÉABILITÉ}

Si l'on arrête la pénétration d'un piézocône (pour reprise de course des vérins de fonçage ou pour arrêt volontaire) la pression interstitielle tend à revenir plus ou moins vite à sa valeur originelle $u_{0}$.

Chacun a eu l'idée d'enregistrer ce retour à zéro de $\Delta \mathrm{u}$ : la figure 14 montre les courbes que nous avions produites à Vienne en 1976. L'annulation d'un $\Delta u$

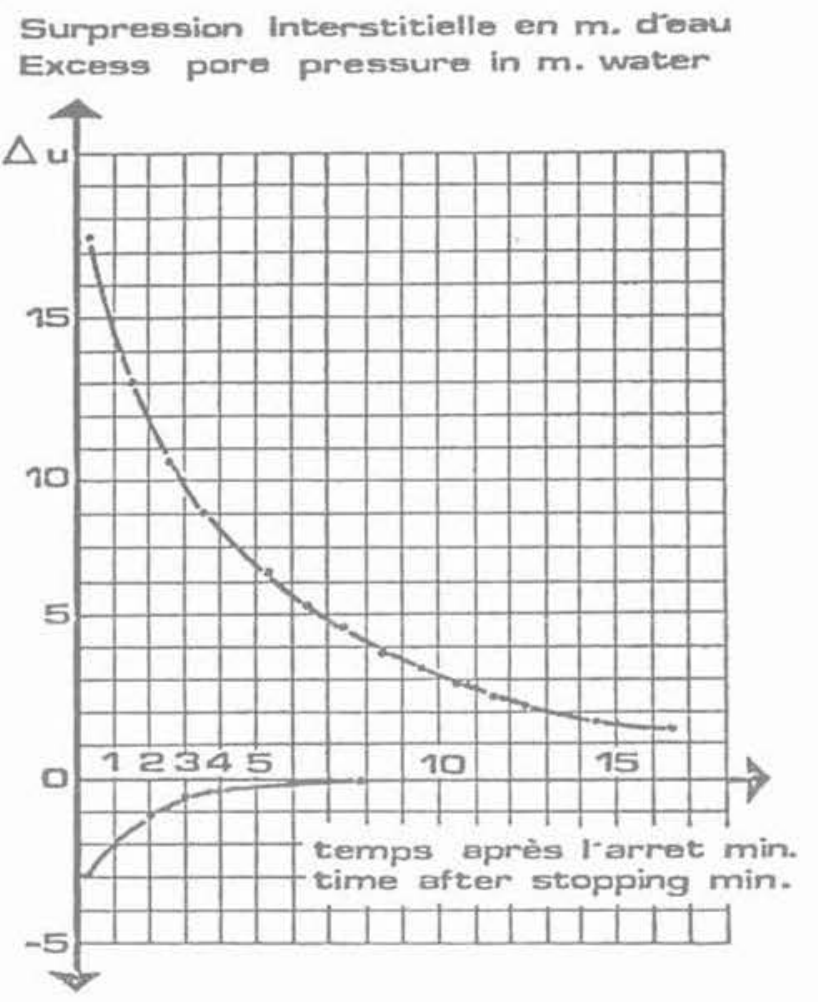

$$
\begin{aligned}
& \text { Fig. 14. - Dissipation de surpression interstitielle } \\
& \text { (1974). } \\
& \text { Fig. 14. - Excess Pore Pressure dissipation (1974). }
\end{aligned}
$$

négatif est bien plus rapide que celle d'un $\Delta u$ positif. La figure 15 due à JONES montre un $\triangle \mathrm{u}$ qui continue à croître deux à trois minutes après l'arrêt du fonçage : ceci est un indice très net d'une saturation incomplète de l'appareil.

La figure 16 montre un essai réalisé en chambre de calibration à l'I.M.G. de Grenoble: on y voit le tracé du $t_{s 0}$, temps nécessaire à la diminution de moitié de $\Delta \mathrm{u}$. On a maintenant pris l'habitude de tracer ces courbes de relaxation de $\Delta \mathrm{u}$ en $\log \mathrm{t}$. 


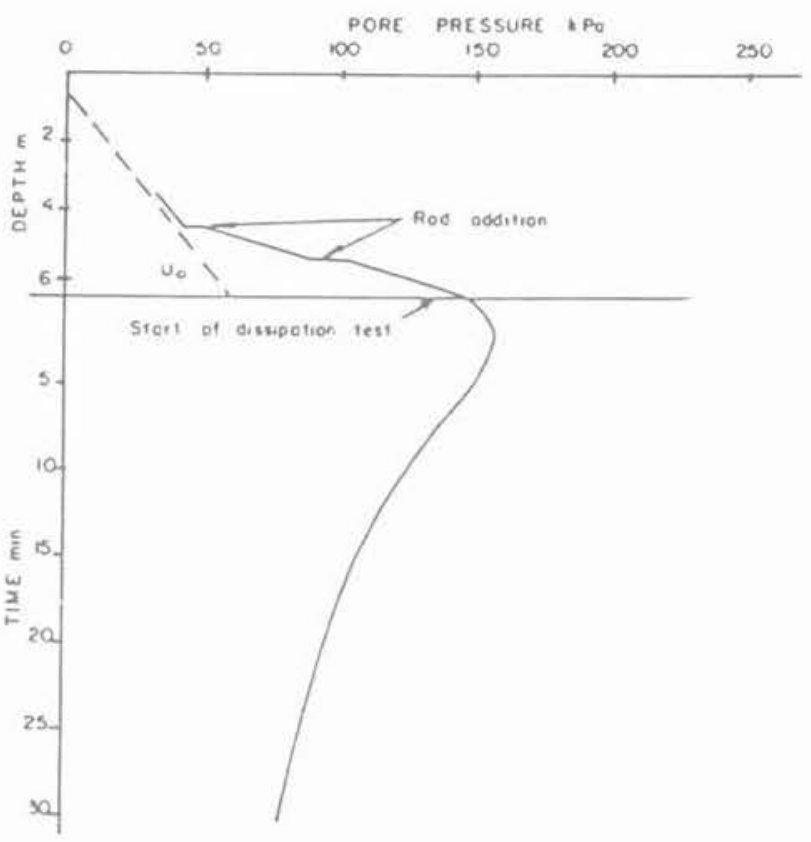

Fig. 15. - Essai de dissipation selon Jones et al. (1981).

Fig. 15 - Dissipation test after Jones and al. (1981).
Sur la figure 3 les petites flèches (à gauche) indiquent les profondeurs oũ des arrêts de fonçage assez longs ont été faits afin d'obtenir les valeurs de $t_{50}$ indiquées dans la colonne de droite. Ces valeurs indiquent les conditions de drainage et de perméabilité du sol. Elles sont différentes selon la position de la bague-filtre.

Lors d'un arrêt pour relaxation de $\Delta u$ faut-il ou non bloquer les tiges? Différents expérimentateurs (DE RUITER, CAMPANELLA) aboutissent à la conclusion suivante:

- si la prise de pression se trouve sur le cône, il est nécessaire de bloquer les tiges sinon il se produit, dès l'arrêt du fonçage dans les argiles, une chute brutale de la pression interstitielle, concommittante avec la baisse de $q_{c}$ :

- si la prise de pression se trouve au-dessus du cône, ce phénomène ne se produit pas d'une façon sensible, il n'est donc pas nécessaire de bloquer les tiges, ce qui est une simplification.

Nous ne retiendrons ici que les résultats pour la.bague située juste au-dessus du cône.

Divers auteurs ont cherché à estimer le coefficient de consolidation du sol à partir de la vitesse de dissipation de $\Delta \mathrm{u}$, à l'aide d'approches théoriques par expansion de cavités.

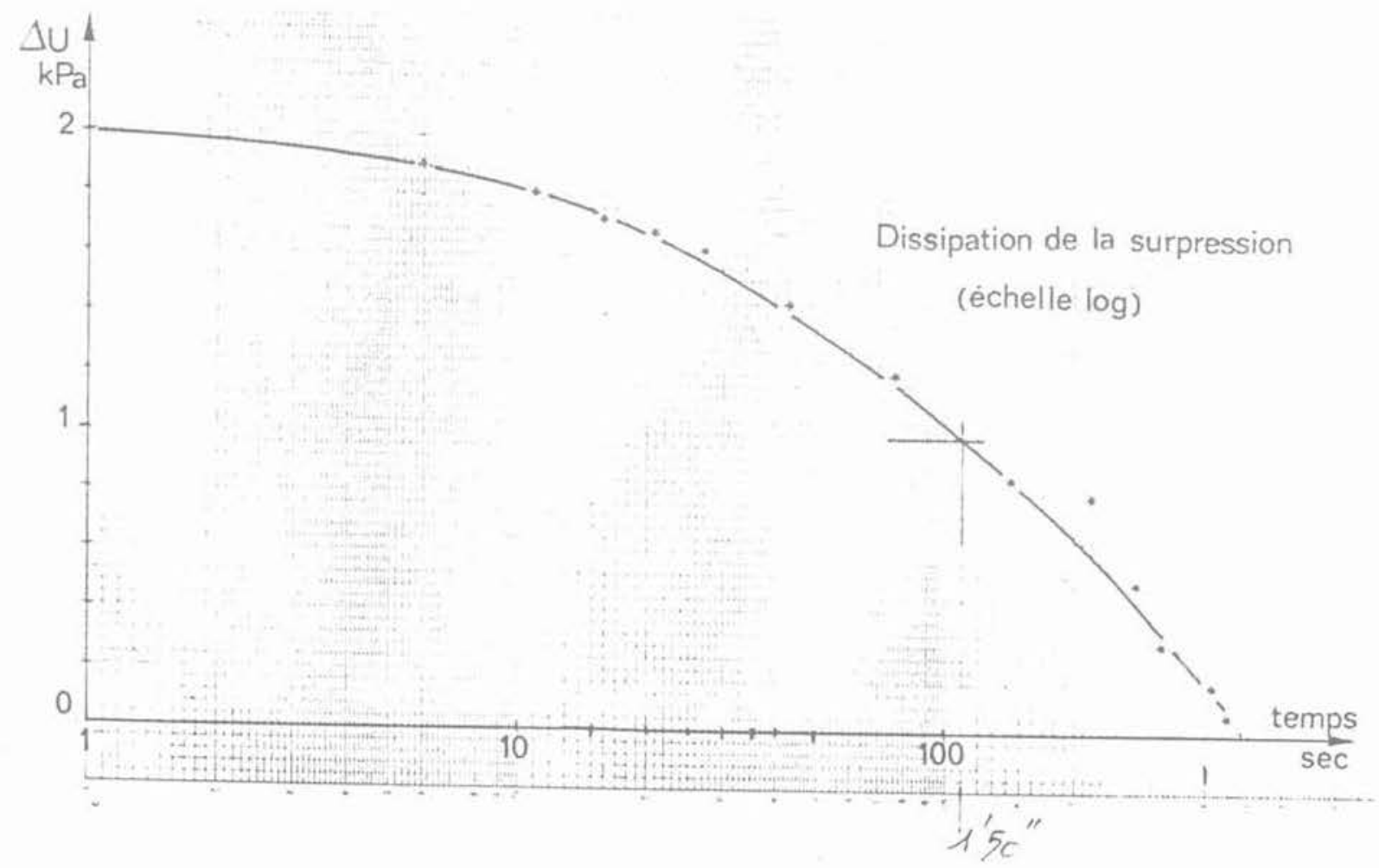

Fig. 16. - Courbe de dissipation en $\log t$.

Fig. 16. - Dissipation graph in $\log t$ 
Différents phénomènes viennent compliquer ces ap. proches. Ce sont en particulier:

- l'effet du remaniement du sol contre le pénétro. mètre;

- limportance relative de la consolidation horizontale et de la verticale;

- la répartition mal connue de la pression interstitielle et des contraintes totales depuis l'extrémité de la pointe du cône jusqu'à la partie cylindrique :

- la position du filtre sous ou au-dessus du cône.

Une approche simplifiée a été donnée par TORS. TENSSON en 1975 résumée sur la figure 17, c'est le
Mais ce coefficient de consolidation, est-ce $\mathrm{C}_{4}$ ou $\mathrm{C}$ ? BALIGH indique qu'au début de la consolidation c'est $\mathrm{C}_{\mathrm{t}}$ qui contrôle la dissipation. Quand $\mathrm{C}_{\mathrm{y}}$ passe de $\mathrm{C}_{\mathrm{b}}$ à $0,1 \mathrm{C}_{\mathrm{h}}$ le facteur temps à $50 \%$ de dissipation $\left(\mathrm{T}_{50}\right)$ augmente de $36 \%$. C'est donc l'erreur qu'on fait sur $\mathrm{C}_{\mathrm{h}}$ si on néglige l'anisotropie. A $36 \%$ près ce n'est déjà pas si mal!

Reste le remaniement contre le pénétromètre. TAVENAS et al. ont trouvé dans de nombreux essais en place sur des argiles canadiennes que la vitesse de dissipation de $\Delta u$ est principalement gouvernée par les caractéristiques de consolidation de l'argile intacte à distance de la sonde. Ainsi $\mathrm{C}$, doit être fonction de la perméabilité $k_{h}$ et du module de déformabilité $\mathrm{M}$ de l'argile intacte.

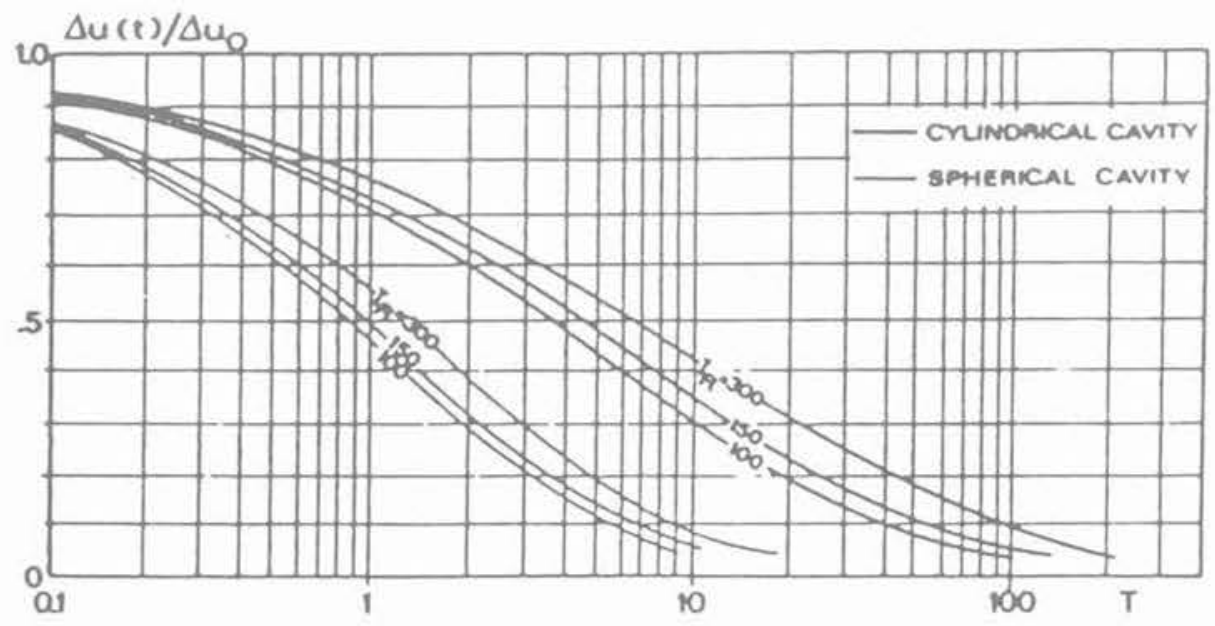

Fig. 17. - Dissipation de la pression interstitielle en fonction du facteur temps (Torstensson 1975).

Fig. 17. - Pore Pressure decay vs. time factor (Torstensson 1975)

faisceau de courbes de droite (cavité cylindrique) qui donne le meilleur résultat pour un piézocône à bague au-dessus du cône. On obtient le facteur temps $T$ pour le degré de consolidation choisi (par exemple $50 \%$ ) en fonction de l'indice de rigidité du sol:

$$
\mathrm{I}_{\mathrm{R}}=\frac{\mathrm{G}_{\mathrm{U}}}{\mathrm{C}_{\mathrm{U}}}
$$

La valeur de $t_{50}$ obtenue sur la courbe de dissipation de $\Delta \mathrm{u}$ permet d'obtenir le coefficient de consolidation par:

$$
C=\frac{T}{t} \times r^{2}
$$

où $r$ est le rayon du piézocône.

CAMPANELLA et al. ont montré que le choix de $50 \%$ de dissipation était très bon tout au moins pour les limons argileux qu'ils ont étudiés.
Pour être bien certain de tout cela, il semble nécessaire de rassembler un maximum de corrélations entre les mesures au piézocône et celles faites au laboratoire, ou mieux, pour les sols suffisamment perméables, celles provenant d'essais de pompage accompagnés de mesures de piézométrie.

Presque tous les auteurs font l'impasse aux sables en disant \& la pénétration à $2 \mathrm{~cm} /$ seconde est suffisamment lente pour qu'on considère qu'il y a drainage total $»$. Mais on s'aperçoit avec les chaînes de mesure modernes à saisie très rapide que $\Delta \mathrm{u}$ n'est pas nul mais souvent négatif si on prend les mesures au-dessus du cône et il est courant de trouver $t_{50}=2$ à 4 secondes pour les sables les plus propres.

Pratiquement $t_{50}$ est voisin de zéro seulement dans les sables et graviers.

Jai mis sur un graphique (figure 18), en fonction de $t_{50}$ (en secondes) les valeurs du coefficient de perméabilité horizontale $\mathrm{k}_{\mathrm{t}}$ en mètre par seconde pour les résultats que je possède. On voit que c'est prometteur, pour les sols normalement consolidés. 


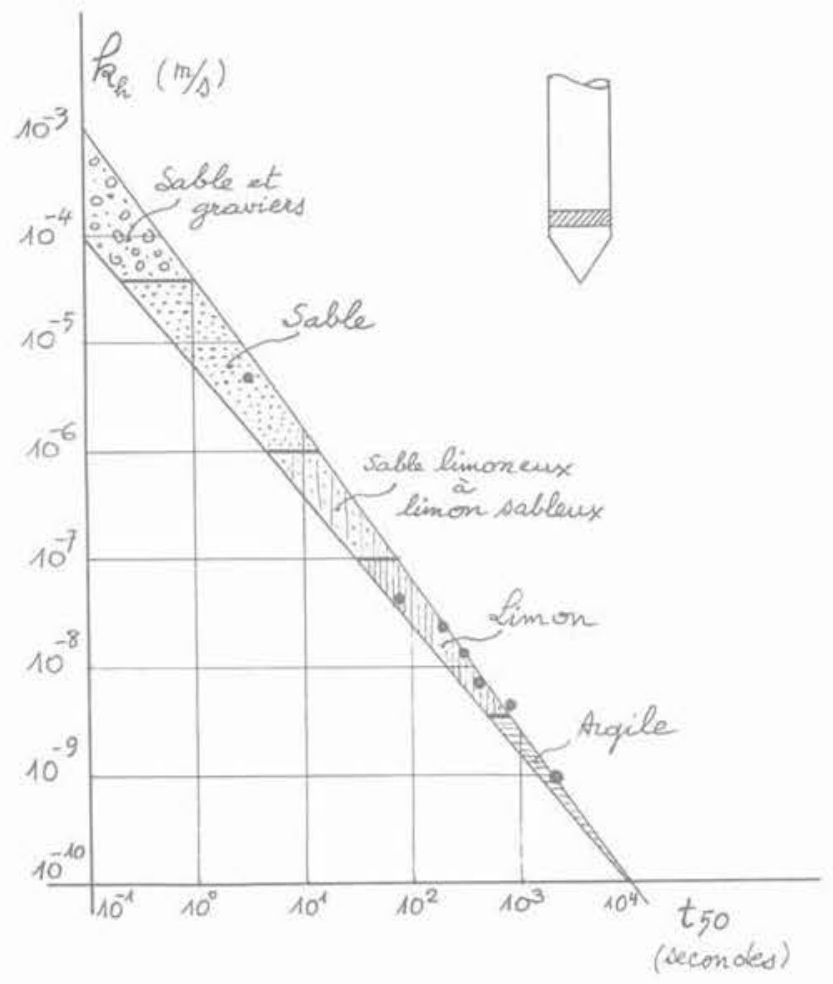

Fig. 18. - Perméabilité horizontale en fonction du $t_{50}$. Fig. 18. - Horizontal permeability vs. $t_{50}$.

Il est donc possible avec le piézocône (en combinaison avec quelques essais de laboratoire) de mieux approcher les problèmes de consolidation dans le temps de couches argileuses surchargées et de juger de l'intérêt économique d'un maillage de drains verticaux.

\section{CONCLUSIONS}

L'enthousiasme de beaucoup de chercheurs montre les potentialités du piézocône.

En plus de l'intérêt des mesures faites au pénétromètre statique:

- courbes continues;

- mesures indépendantes de l'opérateur;

- essai peu coûteux et reproductible;

le piézocône apporte, dans les reconnaissances de sol, les avantages suivants:

- obtention en continu de deux paramètres : $q$ et $u$ qui sont fonction des principales propriétés des couches de sol (avec sur certains appareils, la mesure du frottement sur manchon);

- ces paramètres permettent d'indiquer la stratigraphie avec une précision bien supérieure à celle des forages carottés et, en particulier, de détecter les minces couches (de sable ou d'argile molle) et d'aider à la recherche des surfaces de rupture;

- ces mesures apportent au pénétromètre statique la possibilité d'une analyse en contraintes effectives. Elles doivent aider, dans les années qui viennent, à la prédiction du potentiel de liquéfaction ;

- les essais de dissipation de la pression interstitielle ouvrent la voie à une obtention rapide de profils de perméabilité.

Pour que les résultats des recherches soient comparables entre eux, il est nécessaire que les appareils et les méthodes d'essai soient standardisés (comme l'a été le pénétromètre statique) particulièrement pour les points suivants :

- position, hauteur, épaisseur et perméabilité du filtre ;

- raideur de la membrane du capteur de pression;

- échelle de mesure et précision du capteur;

- méthode de saturation du filtre et de la chambre de mesure;

- méthode de mise en place de lappareil sous la nappe.

\section{BIBLIOGRIAPHIE}

1. BALIGH M.M., LEVADOUX J.N. (1980), Pore Pressure Dissipation after cone penetration. Research Report MITSG 80-13 CAMBRIDGE. Mas.

2. BALIGH M.M., AZZOUZ A.S., WISSA A.E.E., MARTIN R.T., MORRISON M.J. (1981), The Piezocone Penetrometer. A.S.C.E, Geotechnical Division, Symposium on Cone Penetration Testing and Experience, St Louis p. 247-263.

3. CAMPANELLA R.G., GILLESPIE D., ROBERTSON P.K. (1982), Pore Pressures during cone penetration testing. ESOPT 2 Amsterdam, vol. 2, p. 507-512.

4. CANOU J. (1987), Application d'un essai au minipiézocône à l'étude de la liquéfaction des sables. Rapport interne CERMES, ENPC, PARIS.

5. JANBU N., SENNESET K. (1974), Effective stress interpretation of in situ Static Penetration Tests. Proc. ESOPT STOCKHOLM, vol. 2.2, p. 181-193.

6. JONES G.A., VAN ZYL D.J.A. (1981), The piezometric Probe - a useful Investigation Tool. Proc. 10th Int. Conf. S.M.F.E. Stockholm 7/19, p. 489. 496.

7. PAREZ L., BACHELIER M., SECHET B. (1976), Pression interstitielle développée au foncage des pénétromètres. Proc, 6" E.C.S.M.F.E VIENNE (III/ 1.35), 533-538.

8. PAREZ L., BACHELIER M. (1981), C $\mathrm{K}_{\mathrm{v}} \emptyset_{\mathrm{c}}$ déterminés par pénétration statique. Proc. 10th Int. Conf. S.M.F.E. Stockholm 7/32, p. 553-556.

9. SEED H.B., DE ALBA P. (1986), Use of SPT and $C P T$ tests for evaluating the liquefaction resistance of sands. Specialty Conference Use of in situ Tests in Geotechnical Engineering A.S.C.E. Geotechnical Special Publication, $n^{\circ}$ 6, p. 281-302. 
10. SENNESET K., JANBU N., SVAN $\varnothing$ G. (1982), Strength and deformation parameters from cone penetration Tests. ESOPT 2 Amsterdam, vol. 2 , p. $863-870$.

11. SENNESET K., JANBU N. (1984), Shear strenght parameters obtained from static cone penetration tests. A.S.T.M. STP 883 Symposium San Diego.

12. SUGAWARA N., CHIKARAISHI M. (1982), An estimation of $\varnothing^{\prime}$ by using the pore pressure cone penetrometer. Proc. ESOPT 2 AMSTERDAM, vol. 2 , p. 883-888.

13. TAVENAS F., LEROUEIL S., ROY M. (1982), The piezocone test in clay: use and limitations. ESOPT 2 Amsterdam, vol. 2, p. 889-894.

14. TORSTENSSON B.A. (1975), Pore Pressure Sounding Instrument. Proc. A.S.C.E. Specialty Conf. Measurement of Soil Properties, North Carolina State Univ. Raleigh, vol. II, p.48-54. 